\title{
Bone marrow necrosis- an unusual rare finding in multiple myeloma
}

\begin{abstract}
Bone marrow necrosis is a rare but important clinicopathological entity \& is considered challenging to most of the pathologists. It is characterized by destruction of hematopoietic tissue with preservation of the bone. It presents as localized or diffuse generalized process. Many underlying diseases can lead to marrow necrosis, most commonly malignancies and rarely sickle cell disease. The prognosis in patients with bone marrow necrosis is poor. Clinicians must keep a high index of suspicion whenever there is fever, severe bone pain, elevated lactate dehydrogenase, and pancytopenia. Bone marrow biopsy remains the mainstay of the diagnosis We describe here a case of 45-years old patient who presented with sever back pain and was found to have extensive bone marrow necrosis later. The Bone marrow necrosis was due to underlying plasma cell dyscrasia.
\end{abstract}

Keywords: sickle cell disease, vasoactive substances, TNF $\alpha$, CD45, plasma cell dyscrasia
Volume 5 Issue 5 - 2017

\author{
Mariam Al Ghazal, Mohammed Dastagir AH \\ Khan \\ Department of Hematopathology and Cytogenetic, Dammam \\ regional laboratory, Saudi Arabia
}

\begin{abstract}
Correspondence: Mariam Al Ghazal, Department of Hematopathology and Cytogenetic, Dammam regional laboratory, Saudi Arabia, Email dr.m.r.alghazal@hotmail.com
\end{abstract}

Received: August 21, 2017 | Published: December 13, 2017
Abbreviations: HIV, human immunodeficiency virus; G-CSF, granulocyte-colony-stimulating factor; WBC, white blood cell; ESR, erythrocyte sedimentation rate; $\mathrm{CK}$, cytokeratin; $\mathrm{BMN}$, bone marrow necrosis

\section{Introduction}

Bone marrow necrosis is a syndrome first reported by Wade and Stevenson1 in 1941. The typical findings are necrosis of myeloid tissue and medullary stroma leaving an amorphous eosinophilic background, ill-defined necrotic cells, and preserved cortical bone. Bone Marrow Necrosis is usually an unrecognized finding in Routine Bone Marrow Biopsy. According to the extent of Involvement, Bone Marrow Necrosis is classified into Minor (Grade I) involving $<20 \%$ of the BM and Intermediate (Grade II) involving $20-50 \%$ of BM, Sever (Grade III) involving more than $50 \%$ of BM.

The pathophysiology of bone marrow necrosis is still unclear. Failure of the microcirculation accompanied by hypoxemia causes damage to the cells. The toxicity and the release of toxins, cytokines, or vasoactive substances from the injured cells like TNF- $\alpha$ and Interleukin 6 lead to Bone Marrow Endothelial cell Injury with subsequent Micro vascular occlusion play an important role in the pathophysiology of bone marrow necrosis. The incidence of bone marrow necrosis is low. ${ }^{1}$ Etiologies are diverse. Numerous causes of bone marrow necrosis have been identified, including malignancy, radiation/chemotherapy, anorexia, human immunodeficiency virus (HIV)/AIDS, medication, infection, autoimmune disease, disseminated intravascular coagulation, antiphospholipid syndrome and other thrombotic disorders, granulocyte-colony-stimulating factor (G-CSF) exposure, and hemoglobinopathies. ${ }^{2}$

\section{Case presentation}

45years old Saudi male patient who was admitted to neurosurgery department because of severe persistent low back pain, on examination he was pale with no fever, no lymphadenopathy nor organomegaly. Peripheral blood count reveals pancytopenia WBC (white blood cell): $3.79 \times 10^{3} / \mathrm{ul}$ (normal range is $4000-11,000 / \mathrm{ul}$ ) HB: $9.3 \mathrm{~g} / \mathrm{dl}$ (normal range $12.5-18 \mathrm{~g} / \mathrm{dl}$ ) platelets: $32,000 / \mathrm{ul}$ (normal range is $150-450,000 / \mathrm{ul})$. While the Morphology of the peripheral blood shows mild leukoerythroblastic picture (immature myeloid precursors plus normoblasts). There are also $4 \%$ atypical large cells with round nuclei \& abundant light blue cytoplasm. Biochemical profile reveals increased lactic dehydrogenase (LDH): $2126 \mathrm{u} / 1$ (normal range is 100200), increased alkaline phosatase: $1018 \mathrm{u} / 1$ (normal range is 50-136). Albumin level was mildly low $25 \mathrm{~g} / 1$ (normal range is $30-50$ ). Ferritin level is increased $678 \mathrm{ng} / \mathrm{ml}$ (normal range is 16-294).

Total protein is within normal range $71.8 \mathrm{~g} / 1$ (normal range is 62-73). Serum electrolytes including calcium, potassium \& sodium are within normal range for age ESR (erythrocyte sedimentation rate) is 75 (normal range is $0-22 \mathrm{~mm} / \mathrm{hr}$ ). Hepatitis \& HIV human immunodeficiency virus serology are negative. MRI (magnetic resonance imaging) shows Bone marrow infiltration, diffuse low signal intensity of the bone marrow is identified, according to radiologist (Figure 1).

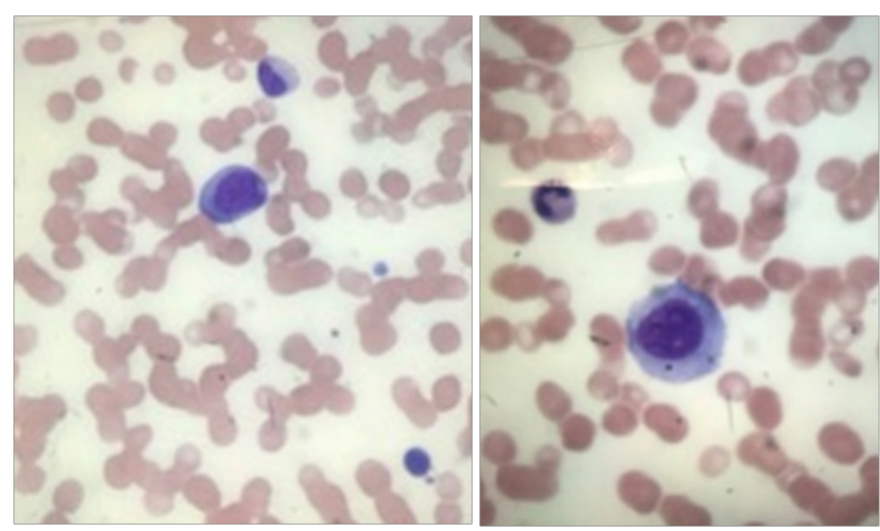

Figure I Mild leukoerythroblastic picture in the left picture, atypical large cell with rounded nuclei \&bluish abundant cytoplasm in the right image. 
The case was referred to our laboratory for flowcytometry to rule out plasma cell dyscrasia. Examination of the bone marrow aspirate shows Pinkish Amorphous background with ghost like cells, accordingly bone marrow Necrosis was suspected Figure 2. Flowcytometry was done on the Bone Marrow sample despite the findings. It showed Majority of the population are present in the CD45 region with Absence of CD34, CD99, CD56 and plasma cell Markers Figure 3, since flowcytometry was not informative atrial of flowcytometry was done from Peripheral blood too and it was also negative for Plasma cell Markers (CD19, CD38, CD138, and CD56) \& immature markers such as CD34 \& CD117. It only showed slight increase in CD56 positive NK cells (27\%) in the lymphocytic gate.

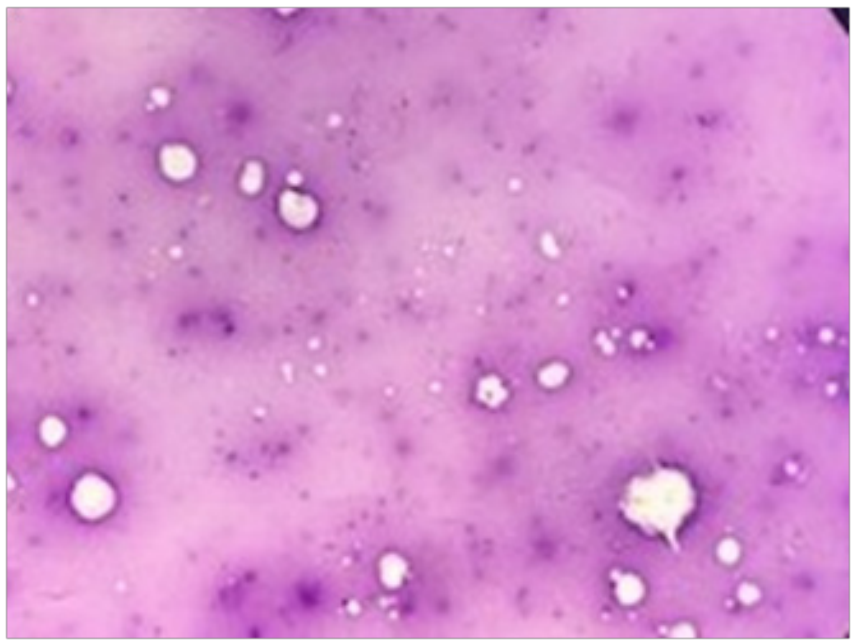

Figure 2 Extensive bone marrow aspirate necrosis with large areas of ghost cells in a background of amorphous eosinophilic material.

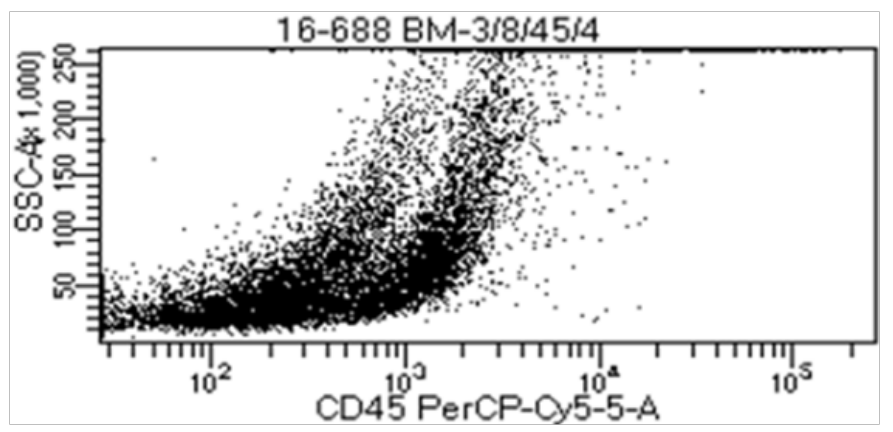

Figure 3 Flowcytometry conducted on the bone marrow aspirate shows that majority of cells are in CD45 negative (lack of viable cells).

One week later another bone marrow aspirate sample came to our laboratory that belongs to the same patient, Bone Marrow morphology shows same findings. background pink staining, many areas of necrosis with ghost like cells, little recovery was noticed in the granulocytes but shows marked changes including many vacuoles and some necrotic changes in nucleus . Flowcytometry done on Bone Marrow Sample for the second time \& still was negative for plasma cells immunophenotype. Trephine biopsy in the second biopsy taken shows Patchy areas of Necrosis comprising of Ghost like cells adjacent to viable cellular elements, at places necrotic debris of nuclei are also seen (Figure 4) .Other areas showed diffuse infiltration by plasma cells (Figure 5). Immunohistochemistry revealed positive staining for CD38, CD138, CD56 and was negative for pan CK (cytokeratin). The patient then was referred to oncology Centre where therapy started; unfortunately he was refractory to it \& passed away 6 months later.

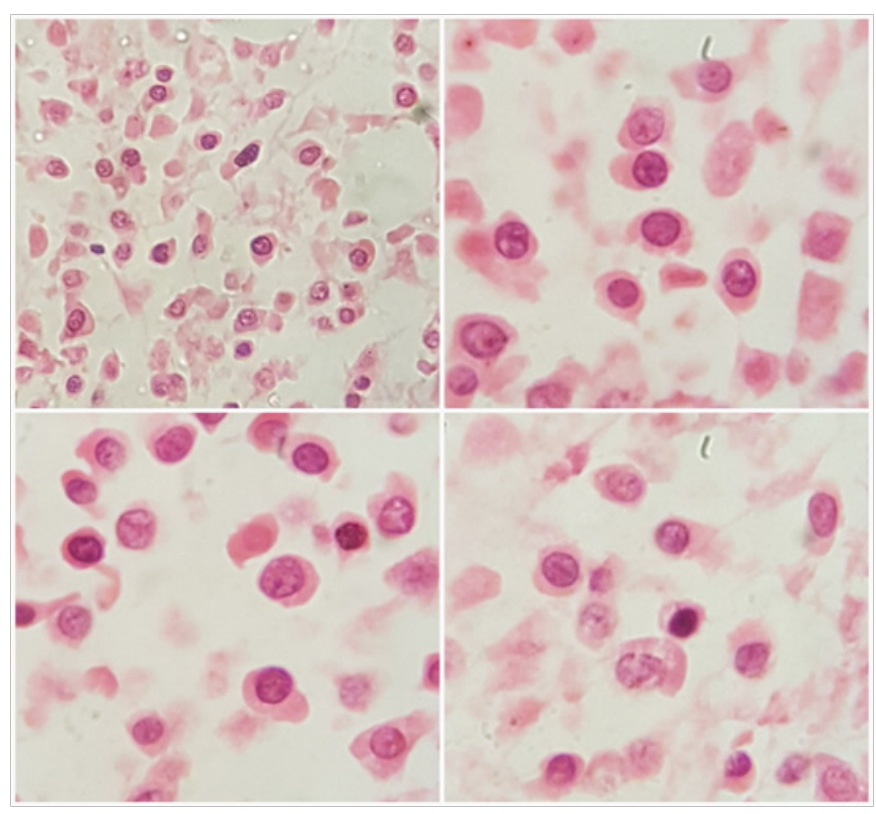

Figure $4 \mathrm{H}$ \& ExI00, stained sections of Second bone marrow biopsy shows the diffuse infiltration of mature plasma cells.

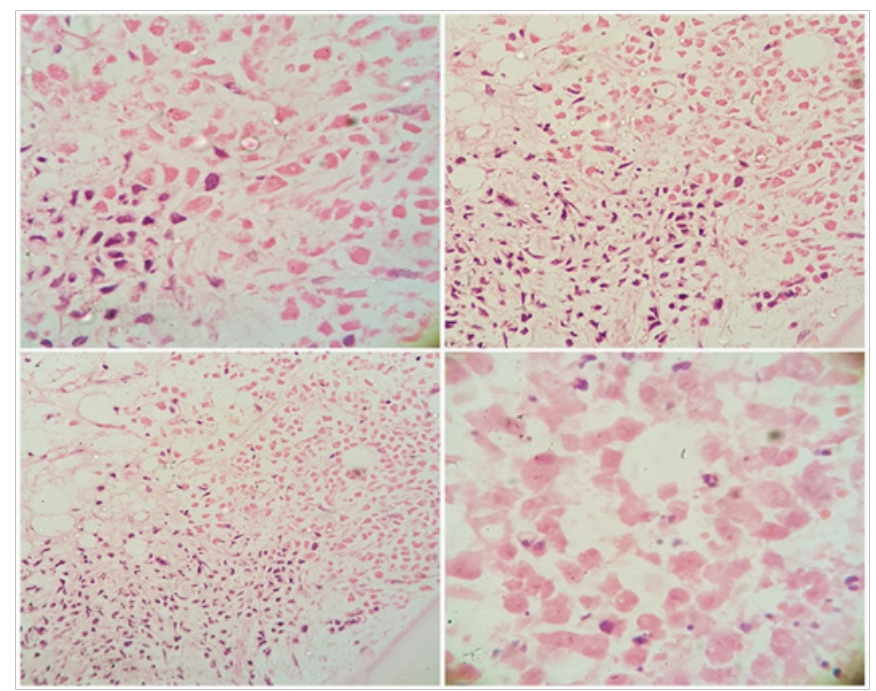

Figure 5 H \& E-x40, stained sections of bone marrow biopsy, the background ghost cells with amorphous pinkish material are noticed.

\section{Discussion}

Bone marrow necrosis (BMN) is regarded as an uncommon entity that is considered as an adverse prognostic factor. Whether it is an independent prognostic marker or whether it is a surrogate marker of underlying disease burden remains unclear. ${ }^{3}$ It is estimated that about 250 to 300 cases of BMN have been reported. ${ }^{4-6}$ Clinically, it is characterized by bone pain, fever, Elevated Alkaline Phosphatase, Markedly Elevated LDH Levels and Peripheral Cytopenias with a Leukoerythroblastic picture and typical findings of Necrosis on Bone Marrow. ${ }^{5-7}$

The morphology of bone marrow necrosis is not easily recognized by many pathologists due to infrequent encountering of the cases. In a 10 years retrospective analysis of cases in our regional laboratory only two cases are diagnosed one was seen in an undiagnosed sickle cell 
disease published in 2017 by Alsafwani et al. ${ }^{8}$ While the second case is this patient. In the patient described herein, Bone Marrow Necrosis developed in the context of Plasma cell dyscrasia. To the best of our knowledge this case is one of few cases of bone marrow necrosis with multiple myeloma background reported in the literature. ${ }^{9}$

In one of the most recent large retrospective analysis published by Wool et al. ${ }^{2}$ approximately $0.3 \%$ of their non-site-directed bone marrow cases showed significant necrosis. Most were secondary to metastatic tumor or hematolymphoid malignancy (together, $90 \%$ of necrosis cases) usually patients with malignancy associated marrow necrosis generally have a poor prognosis (approximately 55\% mortality on the follow up regardless of the tumor type. ${ }^{2}$

Every bone marrow case with necrosis requires morphologic evaluation with examination of the pattern of cellular necrosis, presence of non-necrotic tumor cells or hematolymphoid neoplasm, granulomas, infectious etiologies, and other specific morphologic features. If, despite careful morphologic evaluation, no specific etiology of marrow necrosis can be identified, an initial panel of stains could be considered to evaluate for infectious etiologies and broad categories of neoplasms associated with necrosis in the bone marrow: GMS, AFB, CD3, CD20, CD34, CD117, and pan-cytokeratin (i.e., $\mathrm{AE} 1 / \mathrm{AE} 3+\mathrm{CAM}$ 5.2). In addition, if a repeat bone marrow biopsy is performed, marrow culture and/or flow cytometry could be considered. ${ }^{2}$

Initially the diagnosis of this case was delayed since unfortunately proper morphology and flowcytometry examination didn't help much in reaching the underlying etiology for bone marrow necrosis in the beginning since no evidence for viable hemopoietic or tumor cells were visible by morphology in Bone Marrow, only with repetition of bone marrow the diagnosis was made from the Trephine biopsy findings (diffuse plasma cell infiltration) along with areas of necrosis. Immunohistochemistry conducted with the following antibodies: CD38, CD138, CD56, highlighted the plasma cells faintly. Unfortunately the patient passed away after 6 months of diagnosis as he was resistant to therapy.

This case highlights the importance of considering an extensive search and workup of Bone Marrow Aspirate with Flowcytometry and Morphology and also an Adequate Bone Marrow Biopsy should be examined along with Immunohistochemistry while considering a broad differential diagnosis, if still inconclusive despite the extensive work up a Repeat Bone Marrow Biopsy should always be sought to determine the cause of Bone Marrow Necrosis as the second Biopsy could show viable cells to help reveal the underlying disease. Since diagnosis was not reached initially, we should always ask repeating for bone marrow aspirate \& biopsy to reveal the underlying etiology.

\section{Conclusion}

Bone marrow necrosis $(\mathrm{BMN})$ is a rare antemortem finding seen in wide variety of diseases including hematologic malignancies. Its presence obscures the diagnosis of the underlying diseases but is also indicates poor prognosis. In conclusion, if faced with a patient presenting with pyrexia, bone pain, pancytopenia, with leukoerythroblastic features, bone marrow necrosis must also be considered. The morphology of bone marrow necrosis alone shouldn't distract you from digging to find an underlying possible disease which is crucial in case of masked hematological malignancies. Extensive $\mathrm{BMN}$ associated with hematologic malignancies is almost invariably a fatal complication, however with prompt identification and treatment the prognosis of these patients can be improved.

\section{Acknowledgements}

The authors of this article acknowledged both Dr. Hijjy AlAhmad $\&$ Dr. Jamal Ganim for valuable input of clinical details in this patient.

\section{Conflict of interest}

The authors declare that they have no conflicts of interest.

\section{Author contribution}

Dr. Mariam Al Ghazal, Dr. Mohammedd Dastigir A H Khan were involved in the diagnosis. Dr. Alghazal defined the manuscript while Dr. Khan made the reviews with valuable input. All Authors read and approved the final manuscript.

\section{References}

1. Wang YC, Chang PY, Yao NS. Bone marrow necrosis caused by metastatic colon cancer. J Clin Oncol. 2009;27(23):e48

2. Wool GD, Deucher A. Bone marrow necrosis: ten-year retrospective review of bone marrow biopsy specimens. Am J Clin Pathol. 2015;143(2):201-213.

3. Shapiro R, Rizkalla K, Lam S. Extensive bone marrow necrosis in a case of acute myeloid leukemia transformed from a myeloproliferative neoplasm. Case Rep Oncol. 2015;8(2):345-348.

4. Tamura T, Tasaka T, Fujimoto M, et al. Massive bone marrow necrosis in a patient with chronic myelocytic leukemia following imatinib mesylate therapy. Haematologica. 2004;89(9):ECR32.

5. Janssens AM, Offner FC, Van Hove WZ. Bone marrow necrosis. Cancer. 2000;88(8):1769-1780

6. Rossi D, Ramponi A, Franceschetti S, et al. Bone marrow necrosis complicating posttransplant lymphoproliferative disorder. Leuk Res. 2008;32(5):829-834.

7. Paydas S, Koçak R, Zorludemir S, et al. Bone Marrow necrosis in antiphospholipid syndrome. J Clin Pathol. 1997;50(3):261-262.

8. Alsafwani SA, Al-Saeed A, Bukhamsin R. Extensive bone marrow necrosis: initial presentation in sickle cell anemia-a case report and review of the literature. Case Rep Hematol. 2017;2017:7185604.

9. Sharma R, Gajendra S, Jain S, et al. Multiple myeloma: an unusual cause of extensive bone marrow necrosis. J Clin Diagn Res. 2016;10(6):XL01XL02. 